\title{
Brief Communication: Hair Density and Body Mass in Mammals and the Evolution of Human Hairlessness
}

\author{
Aaron A. Sandel* \\ Department of Anthropology, University of Michigan, Ann Arbor, MI 48109 \\ KEY WORDS body size; skin; allometry; phylogeny
}

\begin{abstract}
Humans are unusual among mammals in appearing hairless. Several hypotheses propose explanations for this phenotype, but few data are available to test these hypotheses. To elucidate the evolutionary history of human "hairlessness," a comparative approach is needed. One previous study on primate hair density concluded that great apes have systematically less dense hair than smaller primates. While there is a negative correlation between body size and hair density, it remains unclear whether great apes have less dense hair than is expected for their body size. To revisit the scaling relationship between hair density and body size in mammals, I compiled data from the literature on 23 primates and 29 nonprimate mammals and conducted Phylogenetic Generalized Least Squares regressions. Among anthropoids, there is a significant negative corre-
\end{abstract}

lation between hair density and body mass. Chimpanzees display the largest residuals, exhibiting less dense hair than is expected for their body size. There is a negative correlation between hair density and body mass among the broader mammalian sample, although the functional significance of this scaling relationship remains to be tested. Results indicate that all primates, and chimpanzees in particular, are relatively hairless compared to other mammals. This suggests that there may have been selective pressures acting on the ancestor of humans and chimpanzees that led to an initial reduction in hair density. To further understand the evolution of human hairlessness, a systematic study of hair density and physiology in a wide range of species is necessary. Am J Phys Anthropol 152:145-150, 2013. (c) 2013 Wiley Periodicals, Inc.

hairs that were greater than $2 \mathrm{~mm}$ in length, which would include terminal hairs and exclude vellus hairs, and found that great apes have lower densities of visible hair than do monkeys. Using these data, Schwartz and Rosenblum (1981) investigated the role of body size on hair density. They found that as the surface area of the body increased, "relative hair density" (RHD: hair$\mathrm{s} / \mathrm{cm}^{2} /$ total surface area of the body) decreased. They proposed that the relationship between body size and hair density represents a thermoregulatory adaptation because larger animals have increased difficulty dissipating heat due to the scaling of volume to surface area. While Schwartz and Rosenblum (1981) did not include data on human body surface area or human hair density in their analysis, they related their anthropoid regression to human hairlessness by predicting that australopiths, with relatively large surface areas, would have had relatively low hair densities. Although they acknowledge that hair may not necessarily impede evaporative cooling (Cena and Monteith, 1975), they alluded to the possibility that it was advantageous for our

\footnotetext{
Additional Supporting Information may be found in the online version of this article.

*Correspondence to: Aaron A. Sandel, Department of Anthropology, University of Michigan, Ann Arbor, MI 48109.
}

E-mail: asandel@umich.edu

Received 30 December 2012; accepted 11 June 2013

DOI: 10.1002/ajpa.22333

Published online 30 July 2013 in Wiley Online Library

(wileyonlinelibrary.com). 
ancestors to reduce their already sparse coats upon moving to open savanna habitats.

This "allometry hypothesis" suggests that there is a connection between body surface area, volume, and hair density, but questions remain. Specifically, it is not clear why Schwartz and Rosenblum (1981) used the variable of "relative hair density" (hairs $/ \mathrm{cm}^{2} /$ total surface area of the body). They estimated total surface area by taking body mass raised to the $2 / 3$ power (Schwartz and Rosenblum, 1981). Dividing hair density by estimated body surface area may obscure the physiological significance of hair density. Within anthropoids, body surface area ranges from $47 \mathrm{~cm}^{2}$ in marmosets to $2,977 \mathrm{~cm}^{2}$ in gorillas (calculated from body mass means from Isler et al., 2008 raised to the $2 / 3$ power). With the denominator differing by several orders of magnitude, relative hair density is heavily skewed by body size making it appear that large animals have sparse pelage and small animals have dense pelage. The "relative hair density" ratio may scale with body mass indicating that body mass itself is not adequately controlled. When plotting "absolute" hair density (hairs $/ \mathrm{cm}^{2}$ ) against body surface area, the negative relationship remains significant (Schwartz and Rosenblum, 1981), but it is not clear that larger primates have less hair than expected for their body size. To determine whether great apes have lower hair density than expected for their body size, it is necessary to conduct an analysis of primate hair density that excludes great apes. While Schwartz and Rosenblum (1981) found a negative relationship between hair density and body size, the functional significance of the scaling relationship remains to be explained.

In this article, I revisit the scaling relationship of hair density and body mass in primates, including additional data on other mammals. Is there a negative correlation between hair density and body mass in nonprimate mammals? If so, do primates, and apes in particular, fit within the general mammalian pattern of hair density and body mass? Adding nonprimate mammals provides data on species that differ considerably in body size, including species with even larger body masses than great apes. If great apes have disproportionally less dense hair than smaller anthropoids, they should exhibit a lower hair density than expected for their body size compared to other primates and mammals. Alternatively, great apes may be in line with other mammals, and the lower hair density of great apes may instead reflect a general mammalian pattern of lower hair density with increasing body size.

\section{METHODS}

To reassess Schwartz and Rosenblum's (1981) allometry hypothesis, I regressed hair density against body mass in 22 anthropoid primates, including humans. Hair density data were extracted from Schultz's (1931) original sample, and included back, scalp, and chest hair densities (although humans were reported as having 0 back hairs $/ \mathrm{cm}^{2}$, so were excluded from the back hair analysis). To make the data comparable, I only included adult individuals and excluded specimens in which the author noted any peculiarities. As many primates exhibit sexual size dimorphism, I used body masses from the literature that best reflected the size of the subjects in the hair density study (Supplementary Material; Inagaki and Hamada, 1985; Smith and Jungers, 1997; Muroyama et al., 2006; Isler et al.,
2008). Schultz (1931) reported the sex of the specimens used in his hair study, so I used body masses of the same sex as the specimen in the hair data and averaged male and female body masses when both sexes were used for hair data.

To extend the analyses to a broader array of animals, I compiled additional data on hair density and body mass for 29 terrestrial nonprimate mammal species from the literature representing eight orders, and one primate, the Japanese macaque (Supplementary Material; hair density: Giacometti and Machida, 1965; Dawson and Brown, 1970; Voipio and Hissa, 1970; Jenkinson and Nay, 1975; McClure and Porter, 1983; Maurel et al., 1986; Korhonen, 1988; Webb and McClure, 1988; Flood et al., 1989; Reynolds, 1993; Fish et al., 2002; Sheriff et al., 2009; body mass: McClure and Porter, 1983; Webb and McClure, 1988; Vleck and Kenagy, 1987 in Lovegrove, 1989; Reynolds, 1993; Sidorovich et al., 1999; Pérez-Barbería and Gordon, 2000; Jones et al., 2009; Sheriff et al., 2009). Mammals exhibit variable types of hair, and different terms are used to describe these different types of hair depending on the taxa being studied. For primates, only terminal hairs were counted, as Schultz only included "visible" hairs that were greater than $2 \mathrm{~mm}$ in length. To make the mammal data comparable to the primate sample of visible (i.e., terminal) hairs, for this analysis, mammal hair densities represent all types of hair that can be counted discretely (combining primary and secondary hairs, or guard hairs and underfur, where applicable). Hair samples were from the dorsal region of most species (including neck, midback, and/or back), although the specific site varied among species (Supplementary Material). Most studies reported counts of hair $/ \mathrm{cm}^{2}$, and for studies that did not, I calculated hairs $/ \mathrm{cm}^{2}$ based on reported hairs/area. Sample sizes ranged from 1 to 33 individuals per species (mode $=1$ individual/species, four species had unknown sample sizes; Supplementary Material). Hair data came from both preserved samples and freshly killed animals. As with the primate analysis, body masses were based on the sex of the individuals used for hair density data whenever possible.

I conducted Phylogenetic Generalized Least Squares (PGLS) regressions in $\mathrm{R}$ using the "caper" package (Orme et al., 2012; R Development Core Team, 2012). Within anthropoids, I conducted two regressions for back hair density: (1) excluding humans, since humans were not in the sample, and (2) excluding great apes. I conducted three regressions for scalp and chest density: (1) excluding humans, (2) excluding great apes, and (3) including all species. For the anthropoid regressions, I used a consensus tree constructed from the 10kTrees website (version 3) (Arnold et al., 2010). To assess whether primates have similar hair densities in different regions, I conducted pairwise correlations of the residuals for scalp, chest, and back hair density (residuals were based on the regressions excluding humans but including other great apes).

For the mammal regression, I used a phylogenetic tree (best estimate tree) constructed from Bininda-Emonds et al. (2007). The parameter $\lambda$ scales the internal branches of the phylogeny and estimates the degree of phylogenetic signal of the residuals of the statistical model (Revell, 2010). Lambda values equal to 0 indicate that the unexplained variation is independent of phylogeny, whereas values equal to 1 indicate that the residuals have a distribution predicted by phylogeny under a 

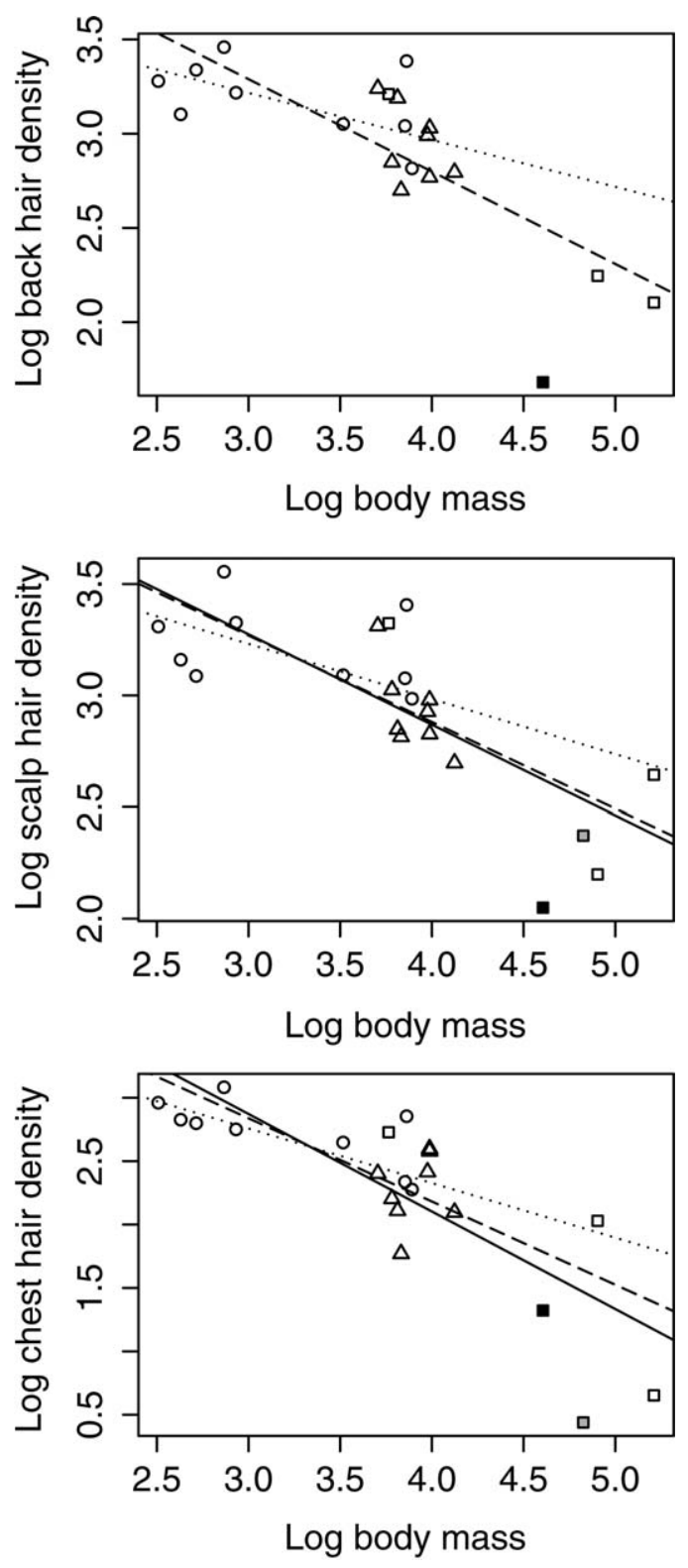

Fig. 1. Log primate back, scalp, and chest hair density (hairs $/ \mathrm{cm}^{2}$ ) against log body mass (g); circles = Ceboidea, triangles $=$ Cercopithecoidea, squares $=$ Hominoidea, black square = chimpanzee, gray square = human; (1) dashed line: regression excluding humans, (2) dotted line: regression excluding great apes, and (3) solid line: regression including all species.

Brownian motion model of evolution. I incorporated the maximum likelihood estimate of $\lambda$ into the analysis.

\section{RESULTS}

Within the 21 anthropoid species (excluding humans), there is a significant negative correlation between body mass and back hair density (log back hair density = $-0.490 \times \log$ body mass $+4.759, \mathrm{SE}=0.092, R^{2}=$ $0.575, P<0.001$; Fig. 1). Chimpanzees display the largest residuals in magnitude, exhibiting less dense hair than is expected for their body size. Excluding great apes from the analysis, there is still a negative correlation between body mass and hair density (Table 1).
There are significant negative correlations between body mass and chest hair density (log chest hair density = $-0.769 \times \log$ body mass $+5.18, \mathrm{SE}=0.127, R^{2}=$ $0.628, P<0.001$ ) and scalp hair density (log scalp hair density $=-0.406 \times \log$ body mass $+4.49, \mathrm{SE}=0.077$, $\left.R^{2}=0.560, P<0.001\right)$. There are still negative correlations between body mass and scalp hair density and chest hair density, respectively, when either humans or all great apes are excluded (Table 1). Human densities for scalp and chest hair clump with great apes, and chimpanzees actually have lower scalp hair densities than humans. Species residuals for different body parts from the regressions excluding humans were correlated (Pearson's $r$, two-tailed test; scalp vs. chest: $r=0.399$, $P=0.073$, scalp vs. back: $r=0.777, P<0.001$, chest vs. back: $r=0.612, P=0.003)$. Thus, even though there are different slopes for different body regions, some primates are generally more or less hairy than expected for their body size. The lambda value for all regressions was 0 , indicating that phylogeny did not strongly influence the regression models.

To investigate whether the negative relationship between hair density and body mass holds true across mammals, I regressed body mass against back hair density in 51 nonhuman mammals. There was a significant negative correlation between body mass and back hair density (log hair density $=-0.34 \times \log$ body mass + 4.77, $\mathrm{SE}=0.0101, R^{2}=0.192, P=0.002$; Fig. 2). Among this wider set of mammals, chimpanzees still exhibit the largest residuals in absolute magnitude. In addition, primates consistently display negative residuals (Table 2). The lambda value was 0.71 , indicating that there is an influence of phylogeny in this broader comparative analysis.

Excluding primates from the analysis, there is still a negative correlation between body mass and hair density among mammals (log back hair density $=-0.272 \times \log$ body mass $+4.507, \mathrm{SE}=0.064, R^{2}=0.375, P<0.001$; Fig. 2). To determine whether primates systematically differ from nonprimates in their hair density, I conducted an analysis of covariance (ANCOVA) with hair density as the dependent variable, taxonomic order (primate vs. nonprimate) as the factor, and body mass added as a covariate. There was no interaction between order (primate vs. nonprimate) and body mass (difference, 2.435; SE, 47; df, $1 ; P=0.125$ ) indicating that the slopes are not significantly different. There was a significant effect of order (primate vs. nonprimate) on hair density (difference, 24.00; SE, 48; df, $1 ; P<0.001$ ), indicating a difference in elevation between the primate and nonprimate regressions.

\section{DISCUSSION}

There is a negative correlation between body mass and hair density in mammals. This is especially apparent among anthropoids, which exhibit a wide range of body sizes (compared to other taxa, such as bovids or rodents), but the negative correlation remains significant among nonprimate mammals. From Schwartz and Rosenblum's analysis, it appears that chimpanzees, gorillas, and orangutans all have similarly low hair densities due to their large body size. Excluding great apes from the primate analysis demonstrates that great apes do have less dense hair than is expected for a primate of their body size. Chimpanzees remain the greatest residual. Given the large difference in body size between great 
TABLE 1. Regression coefficients for back, chest, and scalp density (including all species, excluding humans, and excluding great apes)

\begin{tabular}{lcccc}
\hline Hair density & $\beta$ & SE & Intercept & Adjusted $R^{2}$ \\
\hline Back (no Homo) & -0.490 & 0.092 & 4.759 & 0.575 \\
Back (no great apes) & -0.249 & 0.085 & 3.963 & 0.310 \\
Chest (all primates) & -0.769 & 0.127 & 5.178 & 0.001 \\
Chest (no Homo) & -0.656 & 0.112 & 4.806 & 0.0096 \\
Chest (no great apes) & -0.430 & 0.119 & 4.049 & 0.026 \\
Scalp (all primates) & -0.406 & 0.077 & 4.492 & 0.452 \\
Scalp (no Homo) & -0.388 & 0.082 & 4.433 & 0.560 \\
Scalp (no great apes) & -0.247 & 0.090 & 3.973 & 0.014 \\
\end{tabular}

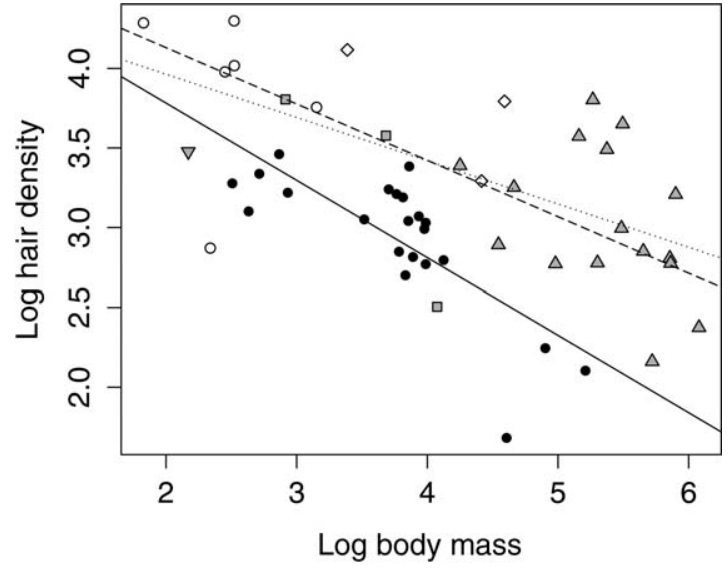

Fig. 2. Log mammal back hair density (hairs $/ \mathrm{cm}^{2}$ ) against log body mass $(\mathrm{g})$; black circles = primates, open circle $=$ Rodentia and Lagomorpha, upward triangle = Artiodactyla, square $=$ Carnivora, downward triangle $=$ Soricomorpha, diamond = Didelphimorphia and Diprotodontia; (1) dashed line: regression including all species, (2) dotted line: nonprimate mammal regression, and (3) solid line: primate regression.

apes and other primates, however, extrapolating great ape hair density from the nongreat ape primate regression should be done with caution. Among all primates, only chimpanzees have especially less dense back hair than expected for their body size. The "relative hair density" score used by Schwartz and Rosenblum reduces the variation among species, obscuring the fact that chimpanzees are an outlier.

What accounts for the overall negative relationship between hair density and body mass? Decreasing hair density may reflect a scaling relationship with increasing total body surface area. Hair follicle formation occurs in embryogenesis, and the absolute number of hair follicles in mammals is set around birth (Szabo, 1967; Mecklenburg et al., 2009). Within an animal's lifetime, hair density is expected to decrease: as surface area increases, interfollicle distances increases. It is possible that, for species with similar hair densities and body sizes at birth, larger mammals will have less dense hair as a product of greater body surface area. Selective pressures involving climate, seasonality, and activity pattern may act on hair density, leading to interspecific variation in the number of hair follicles at birth and throughout an animal's life. While body mass may be an important factor in hair density across mammals, a negative correlation is not necessarily expected and deserves explanation.
TABLE 2. Mammal back hair density (hairs $/ \mathrm{cm}^{2}$ ), body mass (g), and residuals from mammal regression (ordered by residuals)

\begin{tabular}{|c|c|c|c|}
\hline Scientific name & Hairs $/ \mathrm{cm}^{2}$ & $\begin{array}{c}\text { Body } \\
\text { mass (g) }\end{array}$ & $\begin{array}{l}\log \\
\text { residual }\end{array}$ \\
\hline Pan troglodytes & 48 & 40,367 & -1.525 \\
\hline Sigmodon hispidus & 741 & 218 & -1.107 \\
\hline Gorilla beringei & 127 & 162,500 & -0.896 \\
\hline Meles meles & 320 & $11,884.03$ & -0.881 \\
\hline Pongo pygmaeus & 176 & 80,137 & -0.859 \\
\hline Saguinus bicolor & 1,264 & 428 & -0.775 \\
\hline Macaca mulatta & 501 & 6,793 & -0.769 \\
\hline Syncerus caffer & 145 & 526,000 & -0.665 \\
\hline Macaca maura & 588 & 9,720 & -0.647 \\
\hline Callithrix jacchus & 1,896 & 322 & -0.641 \\
\hline Trachypithecus cristatus & 706 & 6,060 & -0.637 \\
\hline Ateles geoffroyi & 652 & 7,780 & -0.635 \\
\hline Papio anubis & 624 & 13,300 & -0.575 \\
\hline Scapanus townsendii & 3,000 & 148 & -0.557 \\
\hline Saimiri sciureus & 1,653 & 855 & -0.557 \\
\hline Cebus capucinus & 1,126 & 3,286 & -0.525 \\
\hline Saguinus geoffroyi & 2,179 & 517 & -0.511 \\
\hline Cercocebus torquatus & 978 & 9,470 & -0.430 \\
\hline Alouatta palliata & 1,099 & 7,150 & -0.420 \\
\hline Nasalis larvatus & 1,072 & 9,730 & -0.386 \\
\hline Macaca fuscata & $1,177.8$ & 8,604 & -0.363 \\
\hline Aotus lemurinus & 2,884 & 734 & -0.337 \\
\hline Litocranius walleri & 780 & 35,000 & -0.335 \\
\hline Bubalus bubalis & 237 & 200,000 & -0.330 \\
\hline Tragelaphus imberbis & 592 & 95,600 & -0.306 \\
\hline Erythrocebus patas & 1,546 & 6,500 & -0.286 \\
\hline Hylobates lar & 1,622 & 5,809 & -0.282 \\
\hline Chlorocebus pygerythrus & 1,739 & 5,071 & -0.272 \\
\hline Oryx beisa & 600 & 200,576 & -0.191 \\
\hline Lagothrix lagotricha & 2,422 & 7,280 & -0.075 \\
\hline Bos gaurus & 596 & 727,500 & -0.004 \\
\hline Bos j & 709 & 450,000 & 0.001 \\
\hline Macropus robustus & 1,960 & $25,978.92$ & 0.021 \\
\hline Mustela putorius & $6,387.5$ & 820.2 & 0.024 \\
\hline Bison bonasus & 641 & 718,000 & 0.026 \\
\hline Rattus no & 9,519 & 282.9 & 0.040 \\
\hline ericanus & $5,699.5$ & 1,418 & 0.056 \\
\hline Vulpes vulpes & 3,780 & $4,820.36$ & 0.058 \\
\hline Eudorcas thomsonii & 2,452 & 17,800 & 0.063 \\
\hline Nanger granti & 1,789 & 46,000 & 0.066 \\
\hline Bos gr & 990 & 306,000 & 0.089 \\
\hline Sciurus vulgaris & 10,425 & 333 & 0.104 \\
\hline Dicrostonyx groenlandicu. & $19,301.5$ & 67.1 & 0.135 \\
\hline Neotoma floridana & 19,900 & 332 & 0.384 \\
\hline Bison bison & 1,616 & 795,300 & 0.443 \\
\hline Didelphis virginiana & 13,076 & $2,442.1$ & 0.497 \\
\hline Kobus ellipsiprymnus & 3,095 & 236,800 & 0.546 \\
\hline Alcelaphus buselaphus & 3,744 & 144,600 & 0.556 \\
\hline Mact & 6,210 & $38,968.39$ & 0.582 \\
\hline Ovibos moschatus & 4,480 & 312,500 & 0.748 \\
\hline Connochaetes taurinus & 6,351 & 184,900 & 0.822 \\
\hline
\end{tabular}


Primates, for their body size, are generally less hairy than other mammals. This may represent a thermoregulatory adaptation. Wheeler $(1984,1985)$ hypothesized that the low hair density in humans was associated with increased sweating capabilities. If the low hair density among primates represents a thermoregulatory adaptation, there should be a negative correlation between eccrine sweat gland density and hair density. There are no comparative data on eccrine sweat gland density in primates, but the distribution of eccrine sweat glands (presence vs. absence in certain body regions) is not consistent with the thermoregulatory predictions (Montagna, 1972; Grant and Hoff, 1975). In sum, the negative relationship between hair density and body mass cannot currently be explained.

Great apes, and chimpanzees in particular, are relatively hairless compared to other mammals. What accounts for the especially low hair density in chimpanzees? The chimpanzee hair pattern may be due to the density of certain types of hair rather than a change in the total number of follicles. While there is a negative correlation between body size and visible hair density in mammals, the human hairless appearance is largely due to the covering of small, unpigmented vellus hairs rather than long, pigmented terminal hairs (Szabo, 1967). In the few studies where it was investigated, vellus hairs account for the hairless appearance of some body regions in other primates, such as the face of gorillas or forehead of stump-tail macaques (Ellis and Montagna, 1962; Montagna et al., 1966). Schultz's hair data on primates only account for visible hairs, and "extremely fine hairs, two millimeters and less in length, were disregarded" (Schultz, 1931:304). Thus, Schultz's sample represents terminal hair density, not total hair follicle density. This leaves open the possibility that chimpanzees, like humans, have a higher proportion of vellus to terminal hairs than other mammals. In other words, chimpanzees and humans may have the density of hair follicles expected for their body size, but a greater number of the follicles produce vellus hairs compared to other primates. To date, I have found no studies reporting vellus hair density in chimpanzees or other apes, which would be key for understanding the evolution of the human hair phenotype.

The similarities in hair among chimpanzees and humans may reflect other similarities in the skin. For example, chimpanzees, gorillas, and humans all share similarities in the distribution of apocrine and eccrine sweat glands (Ellis and Montagna, 1962; Montagna and Yun, 1963). Genetic and physiological studies of hair are needed to investigate the mechanisms underlying the similarities between human and chimpanzee pelage.

The sample of mammal species for which there are data on hair density is still limited. Many species, including chimpanzees, are represented by only one specimen. In addition, individuals in each species are likely to vary in hair density based on age, climate, and other factors. Thus, any conclusions must be made with caution, as hair density values may not accurately represent each species. While I only included hair densities from studies using similar methods, variation in sample collection, preservation of specimens, and other methodological differences may also add error to the analyses. These are problems common to many comparative studies and reflect the current data available on hair density. It will be instructive for future studies to incorporate a larger sample of mammals, including species that appear less hairy, such as elephants, tapirs, and warthogs.

If chimpanzees are indeed relatively hairless compared to other mammals, there may have been a selective pressure acting on the ancestor of humans and chimpanzees that led to an initial reduction in hair density. Current hypotheses for human hair evolution focus on uniquely human traits, such as bipedality or longdistance running. If a reduction in terminal hair density is shared with chimpanzees, we may need to develop hypotheses for human "hairlessness" based on traits that are shared among chimpanzees, bonobos, and humans. Additional studies on chimpanzee skin are needed with adequate sample sizes, focusing on the density of both vellus and terminal hairs. It will also be important to collect data on bonobos to examine whether the pattern of lower hair density is shared among both species of Pan. In addition to hair density in adults, attention to the ontogeny of hair growth is especially important in distinguishing geometric scaling from other adaptations. In studying hair across a wider range of species, we will not only better understand this defining trait of mammals but also shed new light on the peculiar human hair phenotype.

\section{ACKNOWLEDGMENTS}

I am grateful to Charlie Nunn, who provided continual advice and commented on an earlier draft of the manuscript. Thanks also to Kari Allen for methodological advice throughout the formation of this study. Thanks to John Mitani, Michele Rasmussen, Christopher Ruff, and three anonymous reviewers for providing helpful feedback and edits on earlier drafts of the manuscript. This manuscript benefited from the helpful feedback of many faculty and graduate students at Duke University and University of Michigan, especially Matt Cartmill and Chris Wall.

\section{LITERATURE CITED}

Arnold C, Matthew LJ, Nunn CL. 2010. The 10kTrees website: a new online resource for primate phylogeny. Evol Anthropol 19:114-118.

Bininda-Emonds ORP, Cardillo M, Jones KE, MacPhee RDE, Beck RMD, Grenyer R, Price SA, Vos RA, Gittleman JL, Purvis A. 2007. The delayed rise of present-day mammals. Nature 446:507-512.

Cena K, Monteith JL. 1975. Transfer processes in animal coats III. Water vapour diffusion. Proc R Soc B 188:413-423.

Darwin C. 1871. The descent of man, and selection in relation to sex. Princeton, NJ: Princeton University Press.

Dawson TJ, Brown GD. 1970. A comparison of the insulative and reflective properties of the fur of desert kangaroos. Comp Biochem Physiol 37:23-38.

Ellis RA, Montagna W. 1962. The skin of primates. VI. The skin of the gorilla (Gorilla gorilla). Am J Phys Anthropol 20:79-93.

Fish FE, Smelstoys J, Baudinette RV, Reynolds PS. 2002. Fur does not fly, it floats: buoyancy of pelage in semi-aquatic mammals. Aquatic Mamm 28:103-112.

Flood PF, Stalker MJ, Rowell JE. 1989. The hair follicle density and seasonal shedding cycle of the muskox (Ovibos moschatus). Can J Zool 67:1143-1147.

Giacometti L, Machida H. 1965. The skin of the mole (Scapanus townsendii). Anat Rec 153:31-39.

Glass B. 1966. Evolution of hairlessness in man. Science 152 : 294.

Gould SJ. 1977. Ontogeny and phylogeny. Cambridge, MA: Belknap Press of Harvard University Press.

Grant PG, Hoff CJ. 1975. The skin of primates. XLIV. Numerical taxonomy of primate skin. Am J Phys Anthropol 42:151-166. 
Hardy A. 1960. Was man more aquatic in the past? New Sci 7: 642-645.

Inagaki H, Hamada Y. 1985. Differences in hair density of Japanese monkeys (Macaca fuscata fuscata) with locality and age. Primates 26:85-90.

Isler K, Christopher Kirk E, Miller JMA, Albrecht GA, Gelvin BR, Martin RD. 2008. Endocranial volumes of primate species: scaling analyses using a comprehensive and reliable data set. J Hum Evol 55:967-978.

Jablonski N. 2008. Skin: a natural history. Los Angeles: University of California Press.

Jenkinson DM, Nay T. 1975. The sweat glands and hair follicles of different species of bovidae. Aust J Biol Sci 28:55-68.

Jones KE, Bielby J, Cardillo M, Fritz SA, O’Dell J, Orme CDL, Safi K, Sechrest W, Boakes EH, Carbone C, Connolly C, Cutts MJ, Foster JK, Grenyer R, Habib M, Plaster CA, Price SA, Rigby EA, Rist J, Teacher A, Bininda-Emonds ORP, Gittleman JL, Mace GM, Purvis A. 2009. PanTHERIA: a species-level database of life history, ecology, and geography of extant and recently extinct mammals. Ecology 90:2648.

Korhonen H. 1988. Seasonal comparison of body composition and hair coat structure between mink and polecat. Comp Biochem Physiol Part A: Physiology 91:469-473.

Kushlan J. 1985. The vestiary hypothesis of human hair reduction. J Hum Evol 14:29-32.

Lovegrove GG. 1989. The cost of burrowing by the social mole rats (Bathyergidae) Cryptomys damarensis and Heterocephalus glaber: the role of soil moisture. Physiol Zool 62:449-469.

Maurel D, Coutant C, Boissin-Agasse L, Boissin J. 1986. Seasonal moulting patterns in three fur bearing mammals: the European badger (Meles meles L.), the red fox (Vulpes vulpes L.), and the mink (Mustela vison). A morphological and histological study. Can J Zool 64:1757-1764.

McClure PA, Porter WP. 1983. Development of insulation in neonatal cotton rats (Sigmodon hispidus). Physiol Zool 56: $18-32$.

Mecklenburg L, Linek M, Tobin DJ, editors. 2009. Hair loss disorders in domestic animals. Hoboken, NJ: Wiley-Blackwell.

Montagna W. 1972. The skin of nonhuman primates. Am Zool 12:109-124.

Montagna W, Machida H, Perkins E. 1966. The skin of primates. XXVIII. The stump-tail macaque (Macaca speciosa). Am J Phys Anthropol 24:71-85.

Montagna W, Yun JS. 1963. The skin of primates. XV. The skin of the chimpanzee (Pan satyrus). Am J Phys Anthropol 21: $189-203$.

Morgan E. 1982. The aquatic ape: a theory of human evolution. New York: Stein and Day.

Muroyama Y, Kanamori H, Kitahara E. 2006. Seasonal variation and sex differences in the nutritional status in two local populations of wild Japanese macaques. Primates 47: $355-364$.
Orme D, Frecklerton R, Thomas G, Petzoldt T, Fritz S, Isaaec N, Pearse W. 2012. caper: Comparative Analyses of Phylogenetics and Evolution in R. R package version 0.5. Available at: http://CRAN.R-project.org/package $=$ caper

Pagel M, Bodmer W. 2003. A naked ape would have fewer parasites. Proc R Soc B 270:S117-S119.

Pérez-Barbería FJ, Gordon IJ. 2000. Differences in body mass and oral morphology between the sexes in the Artiodactyla: evolutionary relationships with sexual segregation. Evol Ecol Res 2:667-684.

R Development Core Team. 2012. R: a language and environment for statistical computing. Vienna, Austria: R Foundation for Statistical Computing.

Rantala MJ. 1999. Human nakedness: adaptation against ectoparasites? Int J Parasitol 29:1987-1989.

Revell LJ. 2010. Phylogenetic signal and linear regression on species data. Methods Ecol Evol 1:319-329.

Reynolds PS. 1993. Effects of body size and fur on heat loss of collared lemmings, Dicrostonyx groenlandicus. J Mammal 74: 291-303.

Ruxton GD, Wilkinson DM. 2011. Avoidance of overheating and selection for both hair loss and bipedality in hominins. Proc Natl Acad Sci USA 108:20965-20969.

Schultz AH. 1931. The density of hair in primates. Hum Biol 3: 303-321.

Schwartz GG, Rosenblum LA. 1981. Allometry of primate hair density and the evolution of human hairlessness. Am J Phys Anthropol 55:9-12.

Sheriff MJ, Kuchel L, Boutin S, Humphries MM. 2009. Seasonal metabolic acclimization in a northern population of free-ranging snowshoe hares, Lepus americanus. J Mammal 90:761-767.

Sidorovich V, Kruuk H, Macdonal DW. 1999. Body size, and interactions between European and American mink (Mustela lutreola and M. vison) in Eastern Europe. J Zool Lond 248: 521-527.

Smith RJ, Jungers WL. 1997. Body mass in comparative primatology. J Hum Evol 32:523-559.

Szabo G. 1967. The regional anatomy of the human integument with special reference to the distribution of hair follicles, sweat glands and melanocytes. Philos Trans R Soc Lond B 252:447-485

Voipio P, Hissa R. 1970. Correlation with fur density of color polymorphism in Sciurus vulgaris. J Mammal 51:185-187.

Webb DR, McClure PA. 1988. Insulation development in an altricial rodent: Neotoma floridana Thomas. Funct Ecol 2: 237-248.

Wheeler PE. 1984. The evolution of bipedality and loss of functional body hair in hominids. J Hum Evol 13:91-98.

Wheeler PE. 1985. The loss of functional body hair in man: the influence of thermal environment, body form and bipedality. $J$ Hum Evol 14:23-28. 